Pacific Journal of Mathematics

LENGTH-PRESERVING MAPS 


\title{
LENGTH-PRESERVING MAPS
}

\author{
HERBeRT BusemanN
}

1. Introduction. If any two points of the metric space $R$ can be connected by a rectifiable curve then a map of $R$ into a metric space $R^{\prime}$ is length-preserving or equilong, if the length of any curve in $R$ equals that of its image in $R^{\prime}$. An equilong map of $R$ means such a map of $R$ into itself.

Folding a piece of paper repeatedly and in different ways exhibits a great variety of equilong maps of the euclidean plane. The original purpose of the present investigation was to determine all equilong maps which are not too pathological of the euclidean spaces and to find out whether other interesting ${ }^{1}$ spaces admit length preserving maps which are not isometries.

However, equilong maps are connected with other important concepts. If the metric of $R$ is intrinsic, i.e., if the distance of any two points equals the infimum of the lengths of all curves in $R$ connecting these points, then an equilong map $\alpha$ of $R$ into a metric space $R^{\prime}$ does not increase distance: $x y \geqq \alpha x \alpha y$. We denote as shrinkage any map of a metric space $R$ into $R^{\prime}$ satisfying this inequality. Shrinkages which are not equilong enter significantly many branches of mathematics. ${ }^{2}$ In fact, the linguistically preferable term "contraction" was avoided here, because it is widely used in functional analysis for the special shrinkages satisfying $x y \geqq k \alpha x \alpha y$ with $k>1$ (see, for example, [5]). Therefore it seemed worthwhile to study the elementary properties of shrinkages as such.

On the other hand, isometries and local isometries are most important special maps (the latter in the theory of covering spaces) which preserve length. Our results on equilong maps will allow us to weaken the hypotheses in various theorems concerning (local) isometries. It often turns out that the axioms for a $G$-space (see [1]) need not all be satisfied and that a map can be proved to be onto where hitherto this had been assumed.

As to our original aims: we will determine all locally finite equilong maps of the euclidean, hyperbolic, and spherical spaces. The maximal open connected sets on which an equilong map is in-

Received May 22, 1963. This work was supported by a grant from the National Science Foundation.

1 "Interesting" is an essential qualification because there are many spaces with isolated equilong maps.

2 Among the less known applications, the shrinkages of cones on certain surfaces constructed by Resetnyak [6] deserve special mention, because they yield elegant solutions of extremal problems in differential geometry. 
jective are-in contrast to fundamental sets-uniquely determined. They are convex and their closures cover the space. Local finiteness refers to this covering. We thus obtain a division of the space into convex polyhedral regions $D_{1}, D_{2}, \cdots$, from which the equilong map is easily reconstructed.

A locally finite division of the space into convex polyhedral regions $D_{1}, D_{2}, \cdots$ belonging to an equilong map is characterized by the following property which is appealing through its simplicity (although the proof is not simple): the number of $(n-1)$-dimensional faces of the $D_{i}$ having a common $(n-2)$-face is even and if $\delta_{1}, \cdots, \delta_{2 k}$ are the angles between these $(n-1)$-faces in cyclic order then

$$
\delta_{1}+\delta_{3}+\cdots+\delta_{2 k-1}=\delta_{2}+\delta_{4}+\cdots+\delta_{2 k} .
$$

Because the existence of length preserving maps implies homogeneity properties of the space, the most interesting spaces from the point of view of these maps are those which possess large groups of motions. We will see that neither general Minkowski spaces nor the hermitian and quarternion elliptic or hyperbolic spaces admit other locally finite equilong maps than motions.

The initial stages of this work profited from discussions of the author with Professor G. Tallini in Rome.

2. Shrinkages. For purposes of comparison we define an expansion of one metric space $R$ into another, $R^{\prime}$, as a map $\beta$ satisfying $x y \leqq \beta x \beta y$. A shrinkage of $R$ into $R^{\prime}$ is continuous but need not be injective. An expansion $\beta$ is injective, the inverse map $\beta^{-1}$ of $\beta(R)$ on $R$ is a shrinkage and hence continuous, but $\beta$ may be nowhere continuous. For $R=R^{\prime}$ we speak of a shrinkage or an expansion of $R$.

The symbol $(x, y, z)$ means that $x \neq y, y \neq z$ and $x y+y z=x z$. We begin with a simple observation concerning the displacement $x \alpha x$ of a point under a shrinkage. This function is continuous because

$$
|x \alpha x-y \alpha y| \leqq x y+\alpha x \alpha y \leqq 2 x y .
$$

(1) Let $\alpha$ be a shrinkage of $R$. If $(p, x, \alpha p)$ then $x \alpha x \leqq p \alpha p$ with equality only when $p x=\alpha p \alpha x$ and $(x, \alpha p, \alpha x)$.

If $(x, p, \alpha p)$ then $x \alpha x \geqq p \alpha p$ with equality only when $\alpha x \alpha p=x p$ and $(x, \alpha x, \alpha p)$.

For $(p, x, \alpha p)$ gives

$$
x \alpha x \leqq x \alpha p+\alpha p \alpha x \leqq p x+x \alpha p=p \alpha p
$$


and $x \alpha x=p \alpha p$ implies first that $\alpha p \alpha x=p x>0$ and then $(x, \alpha p, \alpha x)$. From $(x, p, \alpha p)$ we conclude

$$
x \alpha x \geqq x \alpha p-\alpha x \alpha p \geqq x \alpha p-x p=p \alpha p>0 .
$$

(2) If $\beta$ is an expansion of $R$ and $(p, \beta p, x)$ then $x \beta x \geqq p \beta p$ with equality only when $\beta p \beta x=p x$ and $(\beta p, x, \beta x)$.

This follows from

$$
x \beta x \geqq \beta p \beta x-\beta p x \geqq p x-\beta p x=p \beta p>0 .
$$

The length of the curve $C: x(t)(a \leqq t \leqq b)$ in a metric space (see [1] or [7]) is denoted by $L(C)$. Obviously,

(3) If $\alpha$ is a shrinkage of $R$ in $R^{\prime}$ and $C$ : $x(t)$ is a rectifiable curve in $R$ and $\alpha C: \alpha x(t)$ is its image then $L(\alpha C) \leqq L(C)$.

If $C$ is the curve $x(t)(a \leqq t \leqq b)$ and we put $x(a)=u, x(b)=v$ then $L(C) \geqq u v$. If the equality sign holds we call $C$ a segment $T(u, v)$ from $u$ to $v$, because $T(u, v)$ is isometric to an interval of length $u v$ on the real axis ([1] or [7]|).

(4) If $\alpha$ is a shrinkage of $R$ in $R^{\prime}$ and $u v=\alpha u \alpha v$ then $\alpha$ maps a segment $T(u, v)$ isometrically on a segment $T(\alpha u, \alpha v)$.

For if $z \in T(u, v)$ then

$$
u v=u z+z v \geqq \alpha u \alpha z+\alpha z \alpha v \geqq \alpha u \alpha v=u v,
$$

whence $u z=\alpha u \alpha z$ and $z v=\alpha z \alpha v$. If $w$ is a fourth point of $T(u, v)$, say on the subsegment $T(u, z)$, then it follows from what we just proved that $w z=\alpha w \alpha z$. This yields:

(5) If $p$ and $q$ are fixed points of a shrinkage of $R$ and if exactly one segment $T(p, q)$ exists then $\alpha$ leaves all points of $T(p, q)$ fixed.

Thus the fixed points of a shrinkage of a euclidean or hyperbolic space form a set which is empty or convex and closed.

$A$ ray in a metric space $R$ is the isometric image of the nonnegative real axis and hence may be represented in the form $p(t)$ $(t \geqq 0)$ with $p\left(t_{1}\right) p\left(t_{2}\right)=\left|t_{1}-t_{2}\right|$. We prove:

(6) Assume that for any two points $x, y$ in $R$ a segment $T(x, y)$ exists and that $(w, x, y)$ and $(x, y, z)$ imply $(w, x, z)$. If $\alpha$ is a 
shrinkage of $R$ and the displacement attains at $p$ a positive minimum (i.e., $p \alpha p=\inf _{x \in R} x \alpha x>0$ ), then $p$ is the origin of a ray which $\alpha$ translates into itself.

Conversely, if $p$ is the origin of a ray which $\alpha$ translates (properly) into itself then $p \alpha p=\inf _{x \in R} x \alpha x$.

Let $T$ be a segment from $p$ to $\alpha p$ and $x$ an interior point of $T$. Put $T_{i}=\alpha^{i} T, p_{i}=\alpha^{i} p, x_{i}=\alpha^{i} x,(i=0,1, \cdots), p \alpha p=\rho$. Then (1) yields $x \alpha x=\rho$ and $\left(x, p_{1}, x_{1}\right)$. Hence by hypothesis $\left(p, p_{1}, x_{1}\right)$. Applying (1) to $x$ and $x_{1}$ we obtain $\left(p_{1}, x_{1}, p_{2}\right)$ hence $\left(p, x_{1}, p_{2}\right)$ and $x x_{1}=$ $p_{1} p_{2}=p p_{1}$. From (4) we conclude that $T_{1}$ is a segment from $p_{1}$ to $p_{2}$ and from $\left(x, p_{1}, x_{1}\right)$ and $\left(p, x_{1}, p_{2}\right)$ that $T \cup T_{1}$ is a $T\left(p, p_{2}\right)$. Continuing in this manner we prove that $\bigcup_{i=0}^{\infty} T_{i}$ is a ray $p(t)(t \geqq 0)$ with $\alpha p(t)=p(t+\rho)$.

Conversely, if $\alpha$ induces the translation $\alpha p(t)=p(t+\rho)(\rho>0)$ of the ray $p(t)$, then for an arbitrary point $x$

$$
p x+n x x_{1}+x p \geqq p x+\sum_{i=1}^{n} x_{i-1} x_{i}+x_{n} p_{n} \geqq p p_{n}=n p p_{1} .
$$

Dividing by $n$ and letting $n \rightarrow \infty$ we obtain $x x_{1} \geqq p p_{1}$.

In order to see how (6) can be applied we prove

(7) Let $R$ be a convex subset of a Banach space with strictly convex spheres and $\alpha$ a shrinkage of $R$. The set $S$ of the points where the displacement xax takes its minimal value is either empty or convex. If the minimum is positive then $S$ is the union of parallel rays and $\alpha$ coincides on $S$ with a translation $\delta$ of the space.

If the minimum is 0 we know from (5) that $S$ is convex. If $p \alpha p=\inf x \alpha x=\rho>0$ then, by (6) $\alpha$ induces a translation on a ray $S_{p}$ with origin $p$. If also $q \alpha q=\rho$ then the says $S_{p}$ and $S_{q}$ are parallel because otherwise $\alpha^{i} p \alpha^{i} q \rightarrow \infty$ for $i \rightarrow \infty$ whereas $\alpha^{i} p \alpha^{i} q \leqq p q$. Therefore $\alpha$ coincides on $S_{p} \cup S_{q}$ with an ordinary translation $\delta$ of the space. This implies $p q=\alpha p \alpha q$ and it follows from (4) that $\alpha$ maps $T(p, q)$ isometrically on $T(\alpha p, \alpha q)$. (Segments are unique because the spheres are strictly convex) Therefore $x \alpha x=p \alpha p$ for $x \in T(p, q)$, whence $T(p, q) \subset S$ and $\alpha x=\delta x$ on $S$.

We add an observation which is of interest in connection with contraction maps (see Introduction).

(8) For any two points $x, y$ of $R$ let a segment $T(x, y)$ exist and let $\alpha$ be a map of $R$ in itself satisfying $x y>\alpha x \alpha y$ for $x \neq y$. If the displacement $x \alpha x$ attains a relative minimum at $f$, then $f$ is 
a fixed point (so that the minimum is absolute) and there is no other fixed point. If $x \alpha x$ attains at $g$ a relative maximum then no point $z$ with $(z, g, \alpha g)$ exists.

If $f \neq \alpha f$ then by (1) any point $x$ on a $T(f, \alpha f)$ would satisfy either $x \alpha x<f \alpha f$ or $f x=\alpha f \alpha x$. Both relations contradict the hypothesis. A second fixed point $f^{\prime}$ satisfies $f f^{\prime}=\alpha f \alpha f^{\prime}$ hence $f^{\prime}=f$. If a point $z$ with $(z, g, \alpha g)$ existed then a segment $T(z, g)$ would contain a point $x$ with $(x, g, \alpha g)$ and arbitrarily small $x g$. But (1) would yield either $x g=\alpha x \alpha g$ or $x \alpha x>g \alpha g$.

If $R$ is a differentiable manifold with a Riemann or Finsler metric then the nonexistence of $z$ means that $g$ lies on the so called cut locus or minimum point locus of $\alpha g$. For compact $R$ both $f$ and $g$ exist. If $R$ is a spherical, or more generally, a spherelike ([1, p 128]) space, then $g$ is also unique because $\alpha g$ is then the antipode to $g$ and any two points and their antipodes have equal distance.

As mentioned in the introduction, the metric of $R$ is called intrinsic if any two points $x, y$ of $R$ can be connected by a curve of finite length and

$$
x y=\inf _{\sigma_{x y}} L\left(C_{x y}\right),
$$

where $C_{x y}$ traverses all curves from $x$ to $y$. If in such a space a curve of minimal length from $x$ to $y$ exists it is a segment $T(x, y)$.

Denote by $S(p, q) \quad(p>0)$ the set of all points $x$ for which $p x<\rho$. If the metric of $R$ is intrinsic and the closure $\bar{S}(p, 2 \rho)$ of $S(p, 2 \rho)$ is compact then a segment $T(x, y)$ exists for any two points $x, y$ in $\bar{S}(p, \rho)$. In particular, if $R$ is compact or finitely compact (which means that all $\bar{S}(p, \rho)$ are compact) then a $T(x, y)$ exists for arbitrary $x, y$. (These facts are implicit in the results of [1] and $[7, \mathrm{p} 142])$

If the metric of $R$ is intrinsic the following converse of (4) holds:

(9) If $\alpha$ is a shrinkage of the space $R$ with an intrinsic metric in the space $R^{\prime}$, moreover $u v=\alpha u \alpha v$ and the image $\alpha C$ of the curve $C$ from $u$ to $v$ is a segment $T(\alpha u, \alpha v)$ then $C$ is a $T(u, v)$ and $\alpha$ maps $C$ isometrically on $\alpha C$.

For if $C$ were not a $T(u, v)$, a curve $C_{0}$ from $u$ to $v$ with $L\left(C_{0}\right)<u v$ would exist and it would follow that

$$
\alpha u \alpha v \leqq L\left(\alpha C_{0}\right) \leqq L\left(C_{0}\right)<u w
$$

(10) Let $R$ be a space with an intrinsic metric and $\alpha$ a con- 
tinuous map of $R$ in $R^{\prime}$. Then $\alpha$ is a shrinkage if and only if, $L(C) \geqq L(\alpha C)$ for any curve $C$ in $R$.

The necessity follows from (3). Let $C$ be a curve from $x$ to $y$ with $L(C)<x y+\varepsilon$; then

$$
x y+\varepsilon>L(C) \geqq L(\alpha C) \geqq \alpha x \alpha y
$$

proves the sufficiency.

An important corollary of (9) was already mentioned:

(11) A length preserving map of a space $R$ with an intrinsic metric into a space $R^{\prime}$ is a shrinkage.

It is clear that a shrinkage of a noncompact space onto itself or of a compact space into itself need not be an isometry. However Freudenthal and Hurewicz proved in [4]:

(12) A shrinkage of a compact space onto itself is a motion. ${ }^{3}$

In conjunction with (11) this yields:

(13) A length preserving map of a compact space with an intrinsic metric onto itself is a motion.

In particular, a locally isometric map of a compact $G$-space onto itself is a motion, a fact which the author proved in [1, $p$ 172] without being aware of the paper [4]. A much more interesting generalization is given in (19).

It may be useful to emphasize that in (13) compactness cannot be replaced by finite compactness. If $\left(x_{1}, \cdots, x_{n-1}, z\right)=(x, z)$ are cartesian coordinates in $E^{n}$ then the relations

$$
\alpha(x, z)=\left\{\begin{array}{l}
(x, z+1) \text { for } z<0 \\
(x, 1-z) \text { for } 0 \leqq z \leqq 1 \\
(x, z-1) \text { for } z>1
\end{array}\right.
$$

define an equilong map of $E^{n}$ on itself which is not a motion.

Since the inverse of an expansion is a shrinkage, (12) is valid also for expansions. However, according to [4] a stronger statement holds:

(14) An expansion of a compact space into itself is a motion.

\footnotetext{
${ }^{3}$ A motion of $R$ is an isometry of $R$ onto itself.
} 


\section{In particular:}

(15) An isometry $\beta$ of a compact space $R$ into itself is a motion.

Since we will apply (15) we give a short proof: If $p \in R-\beta R$ existed then putting $p=p_{0}, p_{i}=\beta^{i} p, R=R_{0}, R_{i}=\beta^{i} R$ we would have (for $k \geqq 1$ ) $p_{i} \in R_{i}-R_{i+1}, p_{i} p_{i+k}=p R_{k} \geqq p R_{1}>0$, and the $p_{i}$ would not have an accumulation point.

From (12) interesting results on special spaces may be obtained. For example:

(16) Let $\alpha$ be a shrinkage of the spherical space $S^{n}$ which is not a motion. Then $\alpha$ has at least one fixed point, maps at least one point on its antipode, and sends at least one pair of antipodes into the same point. $\alpha\left(S^{n}\right)$ lies in a closed hemisphere.

The first two statements follow from well known topological facts, because by (12) the degree of the mapping $\alpha$ is zero. They can also be seen directly: If $\alpha x$ were never antipodal to $x$ then the point $x_{t}$ on $T(x, \alpha x)$ with $x x_{t}=t(x \alpha x) \quad(0 \leqq t \leqq 1)$ would be well defined and depend continuously on $x$, so that $x \rightarrow x_{t}$ would by (12) yield a continuous deformation of $S^{n}$ into a proper subset. If $\delta$ is the antipodal map then $\delta \alpha$ is a shrinkage, hence maps some point $u$ on its antipode, and $\alpha u=u$.

If $\alpha$ is not a motion then it may by (12) be regarded as a map of $S^{n}$ into the $n$-dimensional euclidean space, and it follows from the Theorem of Borsuk and Ulam see [3, p. 337], that $\alpha$ sends at least one pair $a, \alpha^{\prime}$ of antipodal points into the same point $b$.

If $S^{n}$ has curvature 1 , then any point $x$ satisfies $\min \left(x a, x a^{\prime}\right) \leqq \pi / 2$. Therefore $\alpha x b \leqq \pi / 2$ for all $x$ and $\alpha\left(S^{n}\right)$ lies in the hemisphere of $S^{n}$ with center $b$.

3. Locally injective equilong maps. From now on we concentrate on length preserving maps. In particular we study regions in which these maps are injective. At each stage we will make it clear which properties on the spaces enter. The first is domain equivalence. We say:

Domain equivalence holds for two topological spaces $R, R^{\prime}$ if the topological image in $R^{\prime}(R)$ of an open set in $R\left(R^{\prime}\right)$ is open in $R^{\prime}(R)$.

For $R=R^{\prime}$ we follow the classical terminology and speak of domain invariance rather than equivalence. Brouwer's theorem 
states that domain equivalence holds for any two topological manifolds of the same dimension. Therefore all manifolds considered in differential geometry have the property of domain invariance. The finite dimensional, and probably all, $G$-spaces have this property.

Some simple examples will help to elucidate this concept and the facts which we are going to prove. First let $R$ be the set in $E^{3}$ with cartesian coordinates $x, y, z$ consisting of the plane $z=0$ and the line $x=y=0$. The metric of $R$ is here and in the second example the intrinsic metric induced by the euclidean metric in $E^{3}$. The interval $1<z<3, x=y=0$ is isometric to the interval $1<y<3$, $x=z=0$ but the former is the sphere $S((0,0,2), 1)$ and is open, the second is not. Domain invariance does not hold in $R$. The map $\alpha$ of $R$ in itself defined by

$$
\alpha(x, y, 0)=(x, y, 0), \quad \alpha(0,0, t)=(0, t, 0)
$$

preserves length and takes the first interval into the second.

Next take $R$ as the set in $E^{3}$ consisting of the three coordinate axes. The interval $x=y=0,1<z<3$, is isometric to the interval $x=y=0,-1<z<1$. The former is open in $R$, the second is not. The map $\alpha$ which leaves all points on the $x$-axis, on the $y$-axis and the points $z \leqq 1$ on the $z$-axis fixed and maps $(0,0, z)$ with $z>1$ on $(0,0,1-z)$ preserves length and takes the first interval into the second.

For later purposes we point out that in both these spaces motions exist which are not the identity and leave $S((0,0,2), 1)$ pointwise fixed.

As third example we take the ray $t \geqq 0$ with the metric $\left|t_{1}-t_{2}\right|$. The set $0 \leqq t<1$ is isometric to $1 \leqq t<2$, the first is open, the second is not. $t^{\prime}=t+1$ takes the first set into the second and is an isometry, but is not a motion because it does not map $R$ onto itself.

Denoting the restriction of a map $\alpha$ to a set $M$ by $\alpha_{M}$ we say that $\alpha$ is injective on $M$ if $\alpha_{M}$ maps $M$ bijectively on $\alpha(M)$. We prove

(17) Let $R, R^{\prime}$ be locally compact spaces with intrinsic metrics and domain equivalence and $\alpha$ an equilong map of $R$ in $R^{\prime}$ which is injective on the open set $G$. Then for every point $p$ of $G$ a positive $\rho_{p}$ exists such that $\alpha_{G}$ (and hence $\alpha$ ) maps $S\left(p, \rho_{p}\right)$ isometrically on $S\left(\alpha p, \rho_{p}\right)$.

Choose $\delta>0$ such that $\bar{S}(p, \delta)$ is compact and lies in $G$. Then $\alpha_{\theta}$ maps $\bar{S}(p, \delta)$ topologically on $\alpha_{G} \bar{S}(p, \delta)=\alpha \bar{S}(p, \delta)$. It follows from 
domain equivalence that $\alpha S(p, \delta)$ is open, hence $\rho=\rho_{p}>0$ exists such that $\bar{S}\left(p^{\prime}, 2 \rho\right)\left(p^{\prime}=\alpha p=\alpha_{G} p\right)$ lies in $\alpha S(p, \delta)$ and is compact.

For any two points $x^{\prime}, y^{\prime}$ in $\bar{S}\left(p^{\prime}, \rho\right)$ there is a segment $T\left(x^{\prime}, y^{\prime}\right)$ which is contained in $\bar{S}(p, 2 \rho)$. Since $\alpha_{\theta}$ is topological in $\bar{S}(p, \delta)$ and preserves length $\alpha_{\theta}^{-1} T\left(x^{\prime}, y^{\prime}\right)$ is a curve from $x=\alpha_{\theta}^{-1} x^{\prime}$ to $y=\alpha_{\theta}^{-1} y^{\prime}$ of length $L\left(T\left(x^{\prime}, y^{\prime}\right)\right)=x^{\prime} y^{\prime} \geqq x y$, hence by (11) $x^{\prime} y^{\prime}=x y$. In particular $p^{\prime} x^{\prime}=p x$. Therefore $\alpha_{\theta}^{-1} \bar{S}\left(p^{\prime} \rho\right)$ is isometric to $\bar{S}(p, \rho)$ and contained in $\bar{S}(p, \rho)$. It follows from (15) that $\bar{S}(p, \rho)=\alpha_{\theta}^{-1} \bar{S}\left(p^{\prime}, \rho\right)$, which proves (17). Our examples show that (17) is not valid without the hypothesis of domain equivalence.

A map $\beta$ of $R$ in $R^{\prime}$ is locally injective if every point of $R$ has a neighborhood on which $\beta$ is injective. Adhering to the terminology of [1] we do not use the strict analogue to define local isometries but require a little more: The map $\alpha$ of $R$ in $R^{\prime}$ is locally isometric if for every point $p$ of $R$ a positive $\rho_{p}$ exists such that $\alpha$ maps $S\left(p, \rho_{p}\right)$ isometrically on $S\left(\alpha p, \rho_{p}\right)$. We now prove the important fact

(18) THEOREM. If $R$ and $R^{\prime}$ are finitely compact spaces with intrinsic metrics and domain equivalence then a locally injective equilong map of $R$ into $R$ ' is a local isometry of $R$ onto $R^{\prime}$.

Our third example shows that (18) does not hold without the hypothesis of domain equivalence even if $R^{\prime}=R$.

Proposition (17) yields the existence of a positive function $\sigma_{p}$ defined in $R$ such that $\alpha$ maps $\bar{S}\left(p, \rho_{p}\right)$ isometrically on $\bar{S}\left(\alpha p, \rho_{p}\right)$ and hence $S\left(p, \rho_{p}\right)$ on $S\left(\alpha p, \rho_{p}\right)$. Let $\rho(p)$ be the supremum of the numbers $\varepsilon$ for which $\alpha$ maps $\bar{S}(p, \varepsilon)$ isometrically on $\bar{S}(\alpha p, \varepsilon)$. If $\rho(p)=\infty$ then $\alpha$ is an isometry of $R$ on $R^{\prime}$ and $\rho(x)=\infty$ for all $x$ in $R$. If $\rho(p)<\infty$ then $S(p, \varepsilon) \supset S(q, \varepsilon-p q)$ for $p q<\varepsilon$ shows that $|\rho(p)-\rho(q)| \leqq p q$. Therefore $\rho(p)$ is a positive continuous function which has a positive minimum on every $\bar{S}(x, \sigma)$ (which is compact by hypothesis).

Let $q$ be any point of $R$ and $q^{\prime}=\alpha q$. We must prove that for a given point $r^{\prime}$ of $R^{\prime}$ a point $r$ in $R$ exists with $\alpha r=r^{\prime}$. Because $R^{\prime}$ is finitely compact and has an intrinsic metric there is a segment $T^{\prime}$ from $q^{\prime}$ to $r^{\prime}$. Let $\delta>0$ be the minimum of $\rho(p)$ for $p \in \bar{S}\left(q, q^{\prime} r^{\prime}\right)$ and choose $q_{0}^{\prime}=q^{\prime}, q_{2}^{\prime}, \cdots, q_{n}^{\prime}=r^{\prime}$ on $T^{\prime}$ such that $\left(q_{i-1}^{\prime}, q_{i}^{\prime}, q_{i+1}^{\prime}\right)$ and $q_{i-1}^{\prime} q_{i}^{\prime}<\delta$.

Since $\alpha$ maps $\bar{S}(q, \delta)$ isometrically on $\bar{S}\left(q^{\prime}, \delta\right)$ there is a segment $T\left(q, q_{1}\right)$ in $S(q, \delta)$ which $\alpha$ maps isometrically on the subsegment $T\left(q^{\prime}, q_{1}^{\prime}\right)$ of $T^{\prime}$. For the same reason $\alpha$ maps a suitable segment $T\left(q_{1}, q_{2}\right)$ in $S\left(q_{1}, \delta\right)$ on the subsegment $T\left(q_{1}^{\prime}, q_{2}^{\prime}\right)$ of $T^{\prime}$. Thus we arrive at a segment $T\left(q_{n-1}, q_{n}\right)$ mapped on the subsegment $T\left(q_{n-1}^{\prime}, q_{n}^{\prime}\right)=$ 
$T\left(q_{n-1}^{\prime}, r^{\prime}\right)$ of $T^{\prime}$. With $q_{n}=r$ we have $\alpha r=r^{\prime}$. It follows, by the way, from (9) that $\bigcup_{i=1}^{n} T\left(q_{i-1}, q_{i}\right)$ is a $T(q, r)$.

Notice the following application of (13) and (18):

(19) THEOREM. If $R$ is a compact space with an intrinsic metric and domain invariance, then a locally injective length preserving map of $R$ in itself is a motion. ${ }^{4}$

Compactness in (19) cannot be replaced by finite compactness, see [1, p 173], but there are various conditions under which it can. Define $\rho(p)$ as in the proof of (18). We introduce the condition

$$
\inf _{p \in \alpha^{-1}\left(p^{\prime}\right)} \rho(p)>0 \text { for each } p^{\prime} \in \alpha R,
$$

which holds when both $R$ and $R^{\prime}$ are $G$-spaces [1, p 171]. Under the hypotheses of (18) $R$ and $R^{\prime}$ are arcwise and locally arcwise connected and $(*)$ guarantees that $R$ is a covering space of $R^{\prime}$. Therefore we have (compare [1, p 174])

(20) Let $R$ and $R^{\prime}$ be finitely compact spaces with intrinsic metrics and domain equivalence. If the locally injective length preserving map $\alpha$ of $R$ in $R^{\prime}$ satisfies (*) and the fundamental group of $R$ is not isomorphic to a proper subgroup of that of $R^{\prime}$, then $\alpha$ is an isometry of $R$ on $R^{\prime}$

Papers [8], [9] and the last part of [2] deal with conditions which can replace the requirement on the fundamental groups. From (18) and [2] it may be deduced, for example, that a locally. injective equilong map of any (complete) locally Minkowskian space into itself is a motion.

4. Regions of injectivity. A region of injectivity of the map $\beta$ of the space $R$ into the space $R^{\prime}$ is a maximal open connected set on which $\beta$ is injective. If $\beta$ is a locally isometric map of $R$ on $R^{\prime}$ then such a region is what is usually called a fundamental domain (or, depending on the terminology, its interior). We are here interested in regions of injectivity of equilong maps of spaces in themselves. No interesting statements are possible unless the space has special properties, in particular besides domain invariance, one or more of the following three:

${ }^{4}$ The following example shows that domain invariance is necessary: The space consists of the origin 0 and the circles $-2 \cdot 3^{-n}+3^{-n} e^{i \theta}(n=0,1,2, \cdots)$ of the complex plane; the metric is given by arclength. Then $\alpha$ defined by $\alpha(0)=0$ and $\alpha\left(-2 \cdot 3^{-n}+3^{-n} e^{i \theta}\right)$ $=-2 \cdot 3^{-n-1}+3^{-n-1} e^{3 i \theta}$ is locally injective and equilong but is neither onto nor isometric. 
I. A motion of the space which leaves all points of a nonempty open set fixed is the identity.

All spaces considered in differential geometry and all $G$-spaces have this property, [1, p 178]. The first two examples in $\S 4$ are spaces for which I does not hold.

II. If $(x, y, z)$ then the segment $T(x, y)$ is unique. Every point $p$ has a neighborhood $S(p, \delta)$ such the segment $T(x, y)$ is unique for points $x, y$ in $S(p, \delta)$.

Again all the usual spaces of disfferential geometry and all $G$-spaces have this property. A Minkowski space of dimension greater than one does not have it unless its spheres are strictly convex.

III. An isometry of a sphere $S(p, \rho)$ on a sphere $S(q, \rho)(\rho>0)$ can be extended to a motion of the space.

This condition may be trivially satified, namely when no isometric spheres with distinct centers exist and the only isometry of $S(p, \rho)$ is the identy. Such a space satisfies I. If, in addition, domain invariance and property II hold, then our theory implies that its only locally finite (see below) equilong map is the identity.

All simply connected complete Riemann spaces with analytic metrics satisfy II. Particularly interesting among these are the elementary, i.e. the euclidean, hyperbolic, and spherical $(\operatorname{dim}>1)$ spaces, the hermitian or quaternion elliptic and hyperbolic spaces and the elliptic and hyperbolic Cayley planes. Apart from the elliptic spaces these are the only finitely compact $G$-spaces with pairwise mobility, which means: Given four points $x, y, x^{\prime}, y^{\prime}$ with $x y=x^{\prime} y^{\prime}$ then a motion exists which takes $x$ into $x^{\prime}$ and $y$ into $y^{\prime}$ (see [1] for the compact case and [10] for the general case.

Finally we mention that, because of the existence of dilations the Minkowski spaces also satisfy III.

In order not to interrupt our arguments later, we first prove a lemma:

(21) Let $M$ be a closed set with a nonempty interior $M_{i}$ in a finitely compact space which has an intrinsic metric and satisfies II. If $M$ contains with any two points $x, y$ the segment $T(x, y)$ when it is unique, then $M=\bar{M}_{i}$ and with $x$ and $y$ the set $M$ contains at least one, and $M_{i}$ contains all, $T(x, y)$.

Let $p \in M, q \in M_{i}$ and $T=T(p, q)$. We show first that $T-p \subset M_{i}$. 
If $q^{\prime} \in T$ is sufficiently close to, but different from, $q$ then $T\left(q^{\prime}, q\right)$ lies in $M_{i}$, and $T\left(q^{\prime}, q\right), T\left(q^{\prime}, p\right)$ are unique, hence are subsegments of $T$ and lie in $M$. If a point of $T-p$ not in $M_{i}$ existed then traversing $T\left(p, q^{\prime}\right)$ from $q^{\prime}$ towards $p$ we would meet a first point $b \neq p$ not in $M_{i}$. Choose $\delta>0$ such that $T(x, y)$ is unique for $x, y$ in $S(b, \rho)$ and then $u, v$ on $T$ with $(p, u, b), b u<\rho,\left(b, v, q^{\prime}\right)$ and $b v<\rho$. The segments $T(u, x)$ with $x \in M_{i} \cap S(b, \rho)$ lie in $M$, because they are unique and $x, u$ lie in $M$. The set $\bigcup_{x} T(u, x)-u$ is open and contains $b$ which would imply $b \in M_{i}$.

Thus $T-p \subset M_{i}$ whence $p \in \bar{M}_{i}$ and $M=\bar{M}_{i}$. Also, trivially, $T \subset M_{i}$ for $p \in M_{i}$, hence $M_{i}$ contains all $T(p, q)$. If $p, q$ are given points of $M$ then sequences $p_{1}, p_{2}, \cdots$ and $q_{1}, q_{2}, \ldots$ in $M_{i}$ exist tending to $p$ and $q$ respectively. Because $T\left(p_{i}, q_{i}\right) \subset M_{i}$ and $\left\{T\left(p_{i}, q_{i}\right)\right\}$ contains-by finite compactness - a subsequence tending to a segment $T(p, q)$, the latter lies in $M$. Next we observe:

(22) Let $R$ be a metric space which has a countable base and satisfies I and III. Then a bijective locally isometric map $\beta$ of a connected open set $G$ in $R$ on an open set $G^{\prime}$ in $R$ can be extended to a motion of $R$.

By hypothesis each point $p$ of $G$ has a neighborhood $S\left(p, \rho_{p}\right)$ which $\beta$ maps isometrically on $S\left(\beta p, \rho_{p}\right)$. Since $G$ is connected and $R$ has a countable base there is a sequence of points $p_{1}, p_{2}, \ldots$ in $G$ such that $\beta$ maps $S\left(p_{i}, \rho_{i}\right)=S\left(p_{i}, \rho_{p_{i}}\right)$ isometrically on $S\left(\beta_{p_{i}}, \rho_{i}\right)$, $G=\bigcup_{i=1}^{\infty} S\left(p_{i}, \rho_{i}\right)$, and $S\left(p_{i+1}, \rho_{i+1}\right) \cap S_{i} \neq \phi$ where $S_{i}=\bigcup_{i=1}^{i} S\left(p_{i}, \rho_{i}\right)$.

It follows from III that the restriction of $\beta$ to $S\left(p_{i}, \rho_{i}\right)$ is the restriction of a motion $\nu_{i}$ of $R$. It suffices to prove that $\beta=\nu_{1}$ on each $S_{i}$. The assertion is trivial for $S_{1}$. Assume it has been proved for $S_{n}$. Then $\nu_{n+1}=\beta=\nu_{1}$ on $S\left(p_{n+1}, \rho_{n+1}\right) \cap S_{n}$. Since this set is not empty it follows from I that $\nu_{n+1}=\nu_{1}$, in particular $\nu_{n+1}=\nu_{1}=\beta$ on $S_{n+1}$. This result and (17) yield

(23) Let $R$ be a finitely compact space with an intrinsic metric and domain invariance satisfying $I$ and III. If the equilong map $\alpha$ of $R$ is injective on the connected open set $G$ then $\alpha_{\theta}$ can be extended to a motion of $R$.

We add the following:

(24) If (under the hypotheses of (23)) $G$ is a region of injectivity then no boundary point $p$ of $G$ has a neighborhood on which $\alpha$ is injective. Hence two distinct regions of injectivity are disjoint. 
For an indirect proof assume that $\alpha$ is injective on $S(p, \rho)$ $(\rho>0)$. Put $S_{n}=S(p, \rho / n)$. For each $n$ there would be a point $q_{n} \in S_{n}-G \cup S_{n}$ and a point $r_{n} \in G$ such that $\alpha r_{n}=\alpha q_{n}$. Otherwise $\alpha$ would be injective on $G \cup S_{n}$. Because $\alpha$ is injective on $S(p, \rho)$ we have $r_{n} p \geqq \rho$.

Let $q^{\prime} \in S_{n} \cap G$. It follows from (22) that $\alpha q_{n}^{\prime} \alpha r_{n}=q_{n}^{\prime} r_{n}$. On the other hand

$$
\begin{aligned}
2 \rho / n>q_{n} q_{n}^{\prime} & \geqq \alpha q_{n} \alpha q_{n}^{\prime} \geqq \alpha q_{n}^{\prime} \alpha r_{n}-\alpha q_{n} \alpha r_{n}=\alpha q_{n}^{\prime} \alpha r_{n}=q_{n}^{\prime} r_{n} \\
& \geqq r_{n} p-p q_{n}^{\prime}>\rho-\rho / n .
\end{aligned}
$$

If two distinct regions $G, D$ of injectivity were not disjoint then, since neither can be contained in the other, $D$ would contain a boundary point $p$ of $G$ because it is connected.

We now come to an important fact which will enable us in the most interesting cases either to prove the nonexistence of equilong maps which are not motions or to construct all length preserving maps which are not too wild.

(25) THEOREM. Let $R$ be a finitely compact space with an intrinsic metric and domain invariance satisfying $I, I I$ and III. A region of injectivity $D$ of an equilong map of $R$ in itself contains with two points $x, y$ all segments $T(x, y)$, its closure $\bar{D}$ therefore contains with $x, y$ at least one $T(x, y)$. Moreover $\alpha_{\bar{D}}$ is injective and is the restriction of a motion of $R$ to $\bar{D}$.

By (22) there is a motion $\beta$ of $R$ extending $\alpha_{D}$, i.e., $\beta x=\alpha_{D} x=$ $\alpha x$ on $D$. Therefore $\beta^{-1} \alpha$ is an equilong map of $R$ which leaves all points of $D$ fixed. Denote the set of all fixed points of $\beta^{-1} \alpha$ by $M$. Then $M$ is closed, contains $D$ and $\beta^{-1} \alpha x=x$ or $\alpha x=\beta x$ on $M$ hence $\alpha_{M}$ is injective. By (5) $M$ contains $T(x, y)$ when $x, y$ lie in $M$ and $T(x, y)$ is unique. It follows from (21) that the interior $M_{i}$ of $M$ contains with any two points $x, y$ all $T(x, y)$ and that $M=\bar{M}_{i}$. Since $M_{i}$ is connected and contains $D$, moreover $\alpha$ is injective on $M_{i}$ and $D$ is maximal we conclude $M_{i}=D$.

Under the hypotheses of (25) we call $\alpha$ locally finite if $R$ is the union of the closures of the regions of injectivity of $\alpha$ and if this covering of $R$ is locally finite. There will then be a finite or countable number of regions of injectivity $D_{1}, D_{2}, \cdots$. For each $D_{i}$ there is a motion $\beta_{i}$ such that $\beta_{i}^{-1} \alpha$ leaves $D_{i}$ pointwise fixed. Therefore studying the properties of $\alpha$ we may assume that $\alpha$ leaves $D_{1}$ pointwise fixed.

An ellipsoid $R$ in $E^{2}$ with three axes of different lengths and with its intrinsic metric admits a finite number of equilong maps. 
These are generated by the reflections in the three planes $P_{i}$ containing two axes and the following maps $\beta_{i}$ : If $R_{i}, R_{i}^{\prime}$ are the sets on $R$ bounded by $P_{i}$ then $\beta_{i}$ leaves $R_{i}$ pointwise fixed and maps $R_{i}^{\prime}$ on $R_{i}$ by reflection in $P_{i}$. Obviously there is such a variety of spaces possessing isolated equilong maps that neither is it feasible nor would it be worthwhile to determine all spaces admitting equilong maps.

Clearly, the interesting spaces are those which possess large groups of motions. We are now going to examine such spaces for proper equilong maps, that is, length preserving maps which are not motions.

5. Spaces without proper equilong maps. The one dimensional cases, although trivial, are basic. The regions of injectivity of a proper locally finite equilong map $\alpha$ of the real axis are intervals or rays whose endpoints form a discrete set. We may assume that $D_{1}$ has a right endpoint $x_{2}$. Let $D_{2}^{\prime}, D_{3}^{\prime}, \cdots$ be the regions of injectivity to the right of $x_{2}$ in their natural order. Denote the left endpoint of $D_{i}^{\prime}$ by $x_{i}$ and let $R_{i}$ be the reflection $x^{\prime}=2 x-x_{i}$ of the real axis in $x_{i}$. Then for $x>x_{2}$ the map $\alpha$ is given by

$$
\alpha x=R_{2} R_{3} \cdots R_{j} x \text { for } x \in D_{j}^{\prime} .
$$

The procedure is analogous for the $D_{j}$ preceding $D_{1}$ (if any).

If the space is the unitcircle (with length as metric) the construction is similar. Orient the circle. There is a finite number of regions of injectivity for a proper locally finite equilong map $\alpha$ which are arcs and which we call $D_{1}, \cdots, D_{m}$ in the order of the orientation. Denote by $R_{i}$ the reflection in the diameter of the circle passing through the left endpoint of $D_{i}$. Then we still have

$$
\alpha x=R_{2} R_{3} \cdots R_{j} \text { for } x \in D_{j},
$$

but the $D_{j}$ must satisfy two conditions. Their number $m$ must be even. $m=2 k$, otherwise $\alpha$ would be injective on $D_{m} \cup D_{1}$. The right endpoint of $D_{m}$ must stay fixed. If $\delta_{i}$ is the length of $D_{i}$ this yields

$$
\sum_{i=1}^{k} \delta_{2 i-1}=\sum_{i=1}^{k} \delta_{2 i} \quad(=\pi) \text {. }
$$

We want to establish that certain spaces with at least transitive groups of motions do not possess proper equilong maps.

(28) THEOREM. A Minkowski space $R$ ( $\operatorname{dim} R=n \geqq 2)$ with strictly convex spheres ${ }^{5}$ admits a locally finite proper equilong map, if, and

5 The validity of (28) is not contingent upon the strict convexity of the spheres. The latter is equivalent to II and hence necessary for applying (25). 
only if, it possesses the reflection in some hyperplane.

Proof. Assume that $R$ can be reflected in the hyperplane $H$. Then the reflections in all hyperplanes parallel to $H$ also exist. Choose affine coordinates $x_{1}, \cdots, x_{n}$ such that $x_{i}$ is Minkowski length on the $x_{i}$-axis, $H$ is given by $x_{n}=0$ and the line $x_{1}=0, \cdots, x_{n-1}=0$ is normal to $H$ in the Minkowski sense. Then all lines $x_{i}=$ const. $(i=1, \cdots, n-1)$ are normal to all $x_{n}=$ const. Let $x_{n} \rightarrow \beta\left(x_{n}\right)$ be a locally finite equilong map of the $x_{n}$-axis on itself. Then

$$
\left(x_{1}, \cdots, x_{n-1}, x_{n}\right) \rightarrow\left(x_{1}, \cdots, x_{n-1}, \beta\left(x_{n}\right)\right)
$$

defines an equilong map of $R$. Thus a considerable variety of equilong maps can be derived alone from the reflection in $H$.

Conversely, assume $R$ possesses a locally finite proper equilong map $\alpha$. Then at least two regions $D_{1}, D_{2}, \cdots$ of injectivity exist and by (25) all these are convex polyhedral regions. $D_{1}$, which by agreement is left pointwise fixed by $\alpha$, has a boundary point $p$ such that for a suitable $\rho>0$ the sphere $S(p, \rho)$ intersects only $\bar{D}_{1}$ and a single other $\bar{D}_{i}$, say $\bar{D}_{2}$. Then $\alpha$ leaves the disk $S(p, \rho) \cap \bar{D}_{1} \cap \bar{D}_{2}$ fixed and coincides on $S(p, \rho) \cap \bar{D}_{2}$ with a motion $\beta$ of $R$. But $\beta$ must be either the identity, which is impossible because then $D_{1}$ would not be a region of injectivity, or the reflection in the hyperplane carrying $\bar{D}_{1} \cap \bar{D}_{2}$.

Then same can be proved for plane quasihyperbolic geometry (see [1, pp 360, 363, 371, 407]) and also for its higher dimensional analogues. Hyperplanes through arbitrary $n$ points (if $\operatorname{dim} R=n>2$ ) do in general not exist and the result must be interpreted to mean that a hyperplane $H$ and the reflection in $H$ exist.

Next we show that the spaces, which after the elementary and elliptic spaces, have the greatest degree of mobility, do not possess proper equilong maps:

(29) THEOREM. The hermitian elliptic and hyperbolic spaces of (real) dimension greater than 2, the quaternian elliptic and hyperbolic spaces of dimension greater than 4, and the Cayley elliptic and hyperbolic planes ${ }^{6}$ do not possess locally finite length preserving maps other than motions.

None of the spaces in (29) have constant curvature. Therefore, using the result mentioned in the preceding section, it suffices to prove:

${ }^{6}$ The Cayley planes have dimension 16 . The hermitian spaces of dimension 2 and the quarternion spaces of dimension 4 are elementary, see [1]. 
(30) Let $R$ be a finitely compact metric space with an intrinsic metric and a pairwise transitive group of motions. If $R$ possesses a proper equilong map, then $R$ has constant curvature.

Even without the hypothesis that $R$ admits a proper equilong map all spaces in question of dimension less than 4 have constant curvature, so that we may assume that $n=\operatorname{dim} R \geqq 3$. Moreover all spaces satisfy I, II, III.

As in the preceding proof there is a point $p$ on the boundary of $D_{1}$ and a sphere $S(p, \rho)(\rho>0)$ such that $S(p, \rho) \subset \bar{D}_{1} \cup \bar{D}_{2}$. We choose $\rho$ so small that $T(x, y)$ is unique for $x, y \in S(p, \rho)$. Then $S(p, \rho)$ is homeomorphic to $E^{n}$. By (25) the set

$$
N=\bar{D}_{1} \cap \bar{D}_{2} \cap S(p, \rho)
$$

contains with any two points the segment $T(x, y)$ and separates $S(p, \rho)$ into two sets. In the language of differential geometry $N$ is therefore an $(n-1)$-dimensional totally geodesic set. Let $\left(p, d x_{N}\right)$ be the lineal element normal to $N$ at $p$. If any other lineal element $(q, d y)$ is given, then pairwise transitivity guarantees the existence of a motion taking $\left(p, d x_{N}\right)$ into $(q, d y)$. This motion takes $N$ into a totally geodesic set through $q$ and normal to $d y$. It follows from the wellknown theorem of Beltrami, that $R$ has constant curvature.

6. Equilong maps of the elementary spaces. We now study the locally finite equilong maps $\alpha$ of the elementary spaces of dimension $n \geqq 2$ which are not motions. Then there are at least two regions of injectivity. As before we denote these by $D_{1}, D_{2}, \cdots$ and assume that $\alpha$ leaves $D_{1}$ pointwise fixed. By (25) the $D_{i}$ and $\bar{D}_{i}$ are convex sets. We remember that a set in a spherical space $S^{n}$ is called convex if it contains with two points at least one segment. Such a set either lies in a closed hemisphere or is the entire $S^{n}$. Therefore each $D_{i}$ and $D_{i}$ is indeed convex and $D_{i}$ lies in an open hemisphere of $S^{n}$. For brevity we write (D) for the set of the $D_{i}$.

The $r$-faces $(0 \leqq r \leqq n-1)$ of (D) are the $r$-faces of the individual $D_{i}$. Of course, we will call the 0 -faces, and 1-faces also vertices and edges. If the $D_{i}$ are known then $\alpha$ is easily reconstructed. A string $s=\left(D_{1}^{\prime}, \cdots, D_{r}^{\prime}\right)$, where each $D_{j}^{\prime}$ is a $D_{i}$, has the property that $D_{i}^{\prime}$ and $D_{i+1}^{\prime}(j=1, \cdots, r-1)$ have a common $(n-1)$ face. This face is unique if $D_{j}^{\prime} \neq D_{j+1}^{\prime}$. In this case we define $R_{j+1}$ as the reflection of the space in the hyperplane containing $D_{j}^{\prime} \cap D_{j+1}^{\prime}$; or $D_{j}^{\prime}=D_{j+1}^{\prime}$ then $R_{j}$ is the identity map $\varepsilon$ of the space $R$. We define

$$
\beta(s)=R_{2} R_{3} \cdots R_{r}
$$


and complete this definition by putting $\beta\left(D_{i}\right)=\varepsilon$ for strings consisting of a single $D_{i}$.

In terms of strings the map can be described as follows:

Let $s_{k}=\left(D_{1}^{\prime}, \cdots, D_{r}^{\prime}\right)$ be a string from $D_{1}$ to $D_{k}$, i.e., $D_{1}^{\prime}=D_{1}, D_{r}^{\prime}=$ $D_{k}$. Then

$$
\alpha x=\beta\left(s_{k}\right) x \text { for } x \in D_{k} .
$$

This follows from our discussion of the Minkowski case.

(31) implies that $\beta\left(s_{k}\right) x$ is independent of the string from $D_{1}$ to $D_{k}$. Conversely, if for a given locally finite division $D=\left(D_{1}, D_{2}, \cdots\right)$ of an elementary space into convex regions the map $\beta\left(s_{k}\right)$ is independent of the string $s_{k}$ leading from $D_{1}$ to $D_{k}$, then (31) defines an equilong map with the $D_{i}$ as regions of injectivity which leaves $D_{1}$ pointwise fixed.

Let (D) consist of the regions of injectivity of a locally finite equilong map and consider an $m$-face $f_{m}$ of (D) $(0 \leqq m \leqq n-1)$. Take an interior point $w$ of $f_{m}\left(w=f_{0}\right.$ for $\left.m=0\right)$ and a hypersphere $K_{w}^{\prime}$ about $w$ whose radius $\rho>0$ is so small that the ball $w x \leqq \rho$ intersects no other $D_{i}$ than those, $D_{1}^{\prime}, \cdots, D_{k}^{\prime}$, which have $f_{m}$ as a face. Let the $(n-m)$-flat normal to $f_{m}$ at $w$ intersect $K_{w}^{\prime}$ in the $(n-m-1)$-sphere $K_{w}\left(K_{w}=K_{w}^{\prime}\right.$ if $\left.m=0\right)$. The equilong map $\beta$ with (D) as regions of injectivity which leaves $D_{1}^{\prime}$ pointwise fixed induces an equilong map of $K_{w}$ in itself for which the $D_{j}^{\prime} \cap K_{w}$ are the regions of injectivity.

If (D) is an arbitrary locally finite division of the space into (at least two) convex regions, and its $m$-faces are again defined as those of the individual $D_{i}$, we may define $K_{w}$ for a given $f_{m}$ as before and denote by $C\left(f_{m}\right)$ the condition that the $D_{j}^{\prime} \cap K_{w}$ be the regions of injectivity for an equilong map of $K_{w}$. These conditions are essentially independent of the choice of $w$ and $\rho$ in the sense that for different choices leading to $K_{w^{*}}^{*}$ a homothetic transformation will send the $D_{j}^{*} \cap K_{w}$ into the $D_{j}^{\prime} \cap K_{w^{*}}^{*}$.

The conditions $C\left(f_{n-1}\right)$ are trivial, they are satisfied by any (D) and hence will no longer be mentioned. The $C\left(f_{n-2}\right)$ are particularly simple. In this case $\mathrm{K}_{w}$ is a circle and we obtain from (27):

(32) The condition $C\left(f_{n-2}\right)$ means: If $f_{n-1}^{1}, \cdots, f_{n-1}^{l}$ are, in cyclic order, the $(n-1)$-faces of $(D)$ which have $f_{n-2}$ as face then $l$ is even and, if $\delta_{i}$ is the angle between $f_{n-1}^{i}$ and $f_{n-1}^{i+1}\left(f_{n=1}^{l+1}=f_{n}^{1}\right)$ then

$$
\delta_{1}+\delta_{3}+\cdots+\delta_{l-1}=\delta_{2}+\delta_{4}+\cdots+\delta_{l} .
$$

We have shown: 
(33) If $D_{1}, D_{2}, \cdots$ are the regions of injectivity of a locally finite equilong map of an $n$-dimensional $(n>2)$ elementary space, then they satisfy the conditions $C\left(f_{m}\right)$ for all $m$-faces $f_{m}(0 \leqq m \leqq n-2)$.

The converse of (33) also holds, but it is clear that the $C\left(f_{m}\right)$ are not independent. Our discussion of the general case applied to $K_{w}$ contains

If $f_{m}$ is a face of $f_{m+k}(k \geqq 1)$ then $C\left(f_{m}\right)$ implies $C\left(f_{m+k}\right)$.

Thus, if all $f_{m}$ with $m>0$ have vertices, then the $C\left(f_{0}\right)$ yield the remaining $C\left(f_{m}\right)$. It is much more surprising that the simple conditions $C\left(f_{n-2}\right)$ are also sufficient. Although our proof of the converse of (33) will use the $C\left(f_{n-2}\right)$ only, it is of interest to see directly why the $C\left(f_{m}\right)$ with $m<n-2$ are redundant. Therefore we show:

(35) If the converse of (33) holds, then the conditions $C\left(f_{n-2}\right)$ are sufficient.

For $n=2$ the conditions $C\left(f_{0}\right)=C\left(f_{n-2}\right)$ are the only ones in (34), hence (35) is true. Assume (35) has been proved for $n \leqq N-1$.

Let $f_{m}$ be an $m$-face of (D) in $E^{N}(m \leqq N-2)$ and construct a corresponding $(N-m-1)$-sphere $K_{w}\left(w \in f_{m}\right)$ as above. With the previous notations $D_{1}^{\prime}, \cdots, D_{r}^{\prime}$ with $f_{m}$ as face determine regions $D_{i}^{\prime} \cap K_{w}$ on $K_{w}$. An $(m+k)$-face of $\left(D^{\prime}\right)=\left(D_{1}^{\prime}, \cdots, D_{r}^{\prime}\right)$ containing $f_{m}$ intersects $K_{w}$ in a $(k-1)$-face $f_{k-1}^{\prime}$ of $\left(D_{1}^{\prime} \cap K_{w}, \cdots, D_{k}^{\prime} \cap K_{w}\right)$. The condition $C\left(f_{k-1}^{\prime}\right)$ for this set is equivalent to $C\left(f_{m+k}\right)$ for $\left(D^{\prime}\right)$, in particular $C\left(f_{N-m-3}^{\prime}\right)$ to $C\left(f_{N-2}\right)$.

By the induction hypothesis applied to the $(N-m-1)$-sphere $K_{w}$ it follows from the $C\left(f_{N-m-3}^{\prime}\right)$ that the $D_{i}^{\prime} \cap K_{w}$ are the regions of injectivity for an equilong map of $K_{w}$. Therefore $C\left(f_{m}\right)$ holds and it follows from (34) that the $D_{i}$ are the regions of injectivity for an equilong map of $R$. Thus our principal result on elementary spaces is this:

(36) THEOREM. Let $(D)=\left(D_{1}, D_{2}, \cdots\right)$ be a locally finite division of an elementary space $R(\operatorname{dim} R=n \geqq 2)$ into convex regions. Necessary and sufficient for the $D_{i}$ to be the regions of injectivity of an equilong map $\alpha$ of $R$-which is then determined up to motions by (31)-is that every $(n-2)$-face $f_{n-2}$ of any $D_{i}$ satisfy the condition:

If $f_{n-1}^{1}, \cdots, f_{n-1}^{l}$ are the $(n-1)$-faces of the $D_{i}$ having $f_{n-2}$ as face in cyclic order and $\delta_{i}$ is the angle between $f_{n-1}^{i}$ and $f_{n-1}^{i+1}\left(f_{n-1}^{l+1}=f_{n-1}^{1}\right)$ then $l$ is even and

$$
\delta_{1}+\delta_{3}+\cdots+\delta_{l-1}=\delta_{2}+\delta_{4}+\cdots+\delta_{l} .
$$


The proof is the content of the last section.

7. Proof of the main theorem on elementary spaces. If $s=$ $\left(D_{1}^{\prime}, \cdots, D_{r}^{\prime}\right)$ and $s^{\prime}=\left(A_{1}=D_{r}^{\prime}, A_{2}, \cdots, A_{t}\right)$ are strings (always in the given system (D)), then we denote the string $\left(D_{1}^{\prime}, \cdots, D_{r}^{\prime}, A_{1}, \cdots, A_{t}\right)$ by $s \cdot s^{\prime}$. Our conventions on $\beta(s)$ show that then

$$
\beta(s) \beta\left(s^{\prime}\right)=\beta\left(s \cdot s^{\prime}\right) \text {. }
$$

With $s^{-1}$ standing for $\left(D_{r}^{\prime}, D_{r-1}^{\prime}, \cdots, D_{1}^{\prime}\right)$ we have

$$
\beta(s) \beta\left(s^{-1}\right)=\beta\left(s \cdot s^{-1}\right)=\beta\left(s^{-1}\right) \beta(s)=\varepsilon .
$$

The string $s$ is closed if $D_{1}^{\prime}=D_{r}^{\prime}$. If $s$ is closed then $s_{c}=$ $\left(D_{j}^{\prime}, \cdots, D_{r}^{\prime}, D_{2}^{\prime}, \cdots, D_{j-1}^{\prime}, D_{j}^{\prime}\right)(1<j \leqq r)$ is closed and it follows from (37) that

$$
\begin{gathered}
\beta(s)=\varepsilon \text { implies } \beta\left(s_{c}\right)=\varepsilon \text { and conversely. } \\
\text { If } s_{1}=s_{1}^{\prime} s \text { and } s_{2}=s^{-1} s_{2}^{\prime} \text { then } \beta\left(s_{1} s_{2}\right)=\beta\left(s_{1}^{\prime} s_{2}^{\prime}\right) .
\end{gathered}
$$

This is a corollary of (37) and (38). Finally: If $s=s_{1} s_{2} s_{3}$ and $s^{\prime}=$ $s_{1}^{\prime} s_{2}^{-1} s_{3}^{\prime}$ are closed strings and $\beta(s)=\beta\left(s^{\prime}\right)=\varepsilon$ then (39) yields $\beta\left(s_{3} s_{1} s_{2}\right)=$ $\beta\left(s_{2}^{-1} s_{3}^{\prime} s_{1}^{\prime}\right)=\varepsilon$, hence

$$
\beta\left(s_{3} s_{1} s_{3}^{\prime} s_{1}^{\prime}\right)=\beta\left(s_{1} s_{3}^{\prime} s_{1}^{\prime} s_{3}\right)=\varepsilon .
$$

Let $s=\left(D_{1}^{\prime}, \cdots, D_{r}^{\prime}=D_{1}^{\prime}\right)$ be any closed string. A polygonal path $\pi$ belongs to $s$ if it has the following properties: it begins and ends at a point of $D_{1}^{\prime}$. It is the product $\pi=\pi_{1} \cdots \pi_{r}$ of paths $\pi_{i}$ (in the sense of homotopy theory), where $\pi_{i}$ lies, except possibly for its endpoints, in $D_{i}^{\prime}$. The endpoint of $\pi_{i}(i<r)$ and hence the initial point of $\pi_{i+1}$ is an interior point of the common $(n-1)$-face of $D_{i}^{\prime}$ and $D_{i+1}^{\prime}$ if $D_{i}^{\prime} \neq D_{i+1}^{\prime}$ and lies in $D_{i}^{\prime}$ if $D_{i}^{\prime}=D_{i+1}^{\prime}$.

Conversely, let a closed polygonal path $\pi=\pi_{1} \cdots \pi_{r}$ be given such that it begins and ends at a point of $D_{1}^{\prime \prime}$, each $\pi_{i}$ lies, the endpoints possibly excepted, in a $D_{i}^{\prime \prime}$, and if the endpoint of $\pi_{i}(i<r)$ does not lie in $D_{i}^{\prime \prime}$ then $D_{i}^{\prime \prime}$ and $D_{i+1}^{\prime \prime}$ have exactly one common $(n-1)$-face and the point is an interior point of this face. Then $\left(D_{1}^{\prime \prime}, \cdots, D_{r}^{\prime \prime}=\right.$ $\left.D_{1}^{\prime \prime}\right)$ is a closed string, and the only one, to which $\pi$ belongs.

Our rules (37) to (41) contain the following fact:

(42) If $\pi, \pi_{i}$ are polygonal paths belonging to closed strings $s$ and $s_{i}(i=1, \cdots, m)$ and if $\pi \sim \pi_{1} \cdots \pi_{m}$, in the sense that $\pi$ remains after subpaths of the $\pi_{i}$ traversed in opposite senses have been cancelled, then $\beta\left(s_{i}\right)=\varepsilon$ implies $\beta(s)=\varepsilon$.

The considerations of the preceding section reduce the proof of 
(36) to the following:

(43) If $(D)$ satisfies the conditions $C\left(f_{n-2}\right)$ then $\beta(s)=\varepsilon$ for every closed string $s$ in $(D)$.

Let the closed string $s=\left(D_{1}^{\prime}, \cdots, D_{r}^{\prime}=D_{1}^{\prime}\right)$ be given. We may assume that $D_{i}^{\prime} \neq D_{i+1}^{\prime}(1 \leqq i \leqq r-1)$. Then points $p_{i} \in D_{i}^{\prime}\left(p=p_{1}=\right.$ $p_{r}$ ) can be chosen to satisfy the following conditions:

(a) The points $p, p_{i}, p_{i+1}(2 \leqq i \leqq r-2)$ are not collinear (do not lie on a great circle in the spherical case). The segments $T_{i}^{\prime}=$ $T\left(p, p_{i}\right)(2 \leqq i \leqq r-1)$ and $T_{i}=T\left(p_{i}, p_{i+1}\right) \quad\left(1 \leqq i \leqq r, p_{r+1}=p\right)$ are then unique also in the spherical case.

(b) $T_{i}^{\prime}$ or $T_{i}$ have at most one common point with a given $(n-1)$-face of (D) and do not intersect a face of dimension lower than $n-1$. The path $\pi$ formed by the segments $T_{i}$ oriented from $p_{i}$ towards $p_{i+1}$ then belongs to $s$.

(c) The 2 -simplex $S_{i}(2 \leqq i \leqq r-2)$ spanned by $p, p_{i}, p_{i+1}$ does not intersect a face of (D) of dimension less than $n-2$.

There is a finite number (if any) of points $u_{1}, \cdots, u_{t}$ in which $\cup S_{i}$ intersects the $(n-2)$-faces of (D), and the $u_{j}$ are interior points of the $S_{i}$ in which they lie.

Let $q_{m_{i}+1}, q_{m_{i}+2}, \cdots, q_{m_{i+1}}\left(m_{2}=0\right)$ with $q_{1}=p_{2}$ and $q_{m_{i}}=p_{i}$ denote points lying in this order on $T_{i}$. Let $t_{0}=0<t_{1}<\cdots<t_{h}=1$, and denote by $q_{\nu, i}$ the point on the (even in the spherical case unique) segment $T\left(p, q_{i}\right)\left(i=1, \cdots, m_{r-2}\right)$ for which $p q_{\nu, i}=t_{\nu}\left(p q_{i}\right)$.

The points $q_{i}$ and the numbers $t_{\nu}$ can be chosen in such a manner that the $q_{\nu, i}$ have the following properties: No $T\left(q_{\nu, i}, q_{\nu+1, i}\right)$ or $T\left(q_{\nu, i}, q_{\nu, i+1}\right)$ has more than one common point with a given $(n-1)$ face of (D), or intersects a face of (D) of dimension less than $n-1$. Consequently, these segments also avoid the points $u_{j}$. Denote by $Q_{\nu, i}$ the (convex) quadrangle with vertices $q_{\nu, i}, q_{\nu+1, i}, q_{\nu+1, i+1}, q_{i+1, \nu}$ and by $\pi_{\nu, i}$ its boundary with the orientation corresponding to this order of the vertices. If the $q_{i}$ and $t_{\nu}$ are properly chosen then these quadrangles have the following further properties: $Q_{0, i} \subset D_{1}^{\prime}$ for all $i$. For $\nu>0$ a $Q_{\nu, i}$ lies either in one $D_{i}^{\prime}$, or $Q_{\nu, i}$ has common points with exactly two $D_{i}^{\prime}$ which have a common $(n-1)$-face and intersects this $(n-1)$-face in interior points, or, finally, $Q_{\nu, i}$ contains exactly one $u_{j}$ and lies in the union of $D_{i}^{\prime}$ with a common $(n-2)$-face.

Then $\pi_{\nu, i}$ belongs to a closed string $s_{\nu, i}$ and $\pi \sim \prod_{\nu, i} \pi_{\nu, i}$. It is clear that $\beta\left(s_{0, i}\right)=\varepsilon$ and the $\beta\left(s_{\nu, i}\right)=\varepsilon$ for $\nu>0$ in the first two cases because of our rules (37) to (41). In the last case, if $Q_{\nu, i}$ contains $u_{j}$ and $u_{j} \in f_{n-2}^{j}$ then $C\left(f_{n-2}^{j}\right)$ and (37) to (41) guarantee that $\beta\left(s_{\nu, i}\right)=\varepsilon$. It now follows from (42) that $\beta(s)=\varepsilon$. 
In conclusion we point out that this investigation leads to a variety of questions: A first type concerns general shrinkages of special spaces and is exemplified by (16). A second type inquires into the structure of the regions of injectivity of equilong maps of spaces which do not satsify III. A third deals with the equilong maps of special (e.g. the locally elementary) spaces. While it does not seem worth the effort to determine all equilong maps of the elementary spaces, it should be decided, whether the spaces in (29) possess proper equilong maps which are not locally finite.

In addition there are many topics suggested by length preserving maps, for instance, maps of $E^{n}$ into itself which preserve, with a suitable definition, the areas of all two-dimensional surfaces. The locally finite equilong maps of $E^{n}$ have this property for any reasonable area.

\section{REFERENCES}

1. H. Busemann, The Geometry of Geodesics, New York 1955.

2. - Geometries in which the planes minimize area, Annali Mat pur. appl. (IV) 55 (1961), 171-190.

3. D. G. Bourgin, Modern Algebrai Topology, New York 1963.

4. H. Freudenthal and W. Hurrewicz, Dehnungen, Verkürzungen, Isometrien Fund. Math., 26 (1930), 120-122.

5. A. N. Kolmogorov and B. V. Fomin, Functional Analysis vol I, Rochester 1957.

6. Yu. G. Resetnyak, On a special map of a polyhedron (Russian), Mat. Sbornik $\mathbf{5 3}$ (95) (1961), 39-52.

7. W. Rinow, Die innere Geometrie der metrischen Räume, Berlin-Göttingen-Heidelberg 1961.

8. J. Szenthe, Über lokalisometrische Abbildungen von G-Räumen auf sich, Annali Niat. pur. appl. (IV) $\mathbf{5 5}$ (1961) 37-46.

9. - Über metrische Räume, deren lokalisometrische Abbildungen Isometrien sind, Acta Math. Acad. Sci. Hungar., 13 (1962), 443-441.

10. J. Tits, Sur certaines classes d'espaces homogènes de groupes de Lie, Mem. Acad. royale Belgique, $\mathrm{Cl}$. Sciences, vol in $8^{\circ}, \mathbf{2 9}$ (1955), fasc. 3. 



\title{
PACIFIC JOURNAL OF MATHEMATICS
}

\author{
EDITORS
}

\author{
Robert Osserman \\ Stanford University \\ Stanford, California \\ M. G. Arsove \\ University of Washington \\ Seattle 5, Washington
}

\author{
J. Dugundji \\ University of Southern California \\ Los Angeles 7, California \\ Lowell J. Paige \\ University of California \\ Los Angeles 24, California
}

\section{ASSOCIATE EDITORS}
E. F. BECKENBACH
B. H. NEUMANN
F. WOLF
K. YOSHIDA

\section{SUPPORTING INSTITUTIONS}

\author{
UNIVERSITY OF BRITISH COLUMBIA \\ CALIFORNIA INSTITUTE OF TECHNOLOGY \\ UNIVERSITY OF CALIFORNIA \\ MONTANA STATE UNIVERSITY \\ UNIVERSITY OF NEVADA \\ NEW MEXICO STATE UNIVERSITY \\ OREGON STATE UNIVERSITY \\ UNIVERSITY OF OREGON \\ OSAKA UNIVERSITY \\ UNIVERSITY OF SOUTHERN CALIFORNIA
}

\author{
STANFORD UNIVERSITY \\ UNIVERSITY OF TOKYO \\ UNIVERSITY OF UTAH \\ WASHINGTON STATE UNIVERSITY \\ UNIVERSITY OF WASHINGTON \\ AMERICAN MATHEMATICAL SOCIETY \\ CALIFORNIA RESEARCH CORPORATION \\ SPACE TECHNOLOGY LABORATORIES \\ NAVAL ORDNANCE TEST STATION
}

Mathematical papers intended for publication in the Pacific Journal of Mathematics should by typewritten (double spaced), and on submission, must be accompanied by a separate author's résumé. Manuscripts may be sent to any one of the four editors. All other communications to the editors should be addressed to the managing editor, L. J. Paige at the University of California, Los Angeles 24, California.

50 reprints per author of each article are furnished free of charge; additional copies may be obtained at cost in multiples of 50 .

The Pacific Journal of Mathematics is published quarterly, in March, June, September, and December. Effective with Volume 13 the price per volume (4 numbers) is $\$ 18.00$; single issues, $\$ 5.00$. Special price for current issues to individual faculty members of supporting institutions and to individual members of the American Mathematical Society: $\$ 8.00$ per volume; single issues $\$ 2.50$. Back numbers are available.

Subscriptions, orders for back numbers, and changes of address should be sent to Pacific Journal of Mathematics, 103 Highland Boulevard, Berkeley 8, California.

Printed at Kokusai Bunken Insatsusha (International Academic Printing Co., Ltd.), No. 6, 2-chome, Fujimi-cho, Chiyoda-ku, Tokyo, Japan.

PUBLISHED BY PACIFIC JOURNAL OF MATHEMATICS, A NON-PROFIT CORPORATION

The Supporting Institutions listed above contribute to the cost of publication of this Journal, but they are not owners or publishers and have no responsibility for its content or policies. 


\section{Pacific Journal of Mathematics}

\section{Vol. 14, No. $2 \quad$ June, 1964}

Tom M. (Mike) Apostol and Herbert S. Zuckerman, On the functional equation $F(m n) F((m, n))=F(m) F(n) f((m, n)) \ldots \ldots \ldots \ldots \ldots \ldots \ldots \ldots \ldots \ldots \ldots$

Reinhold Baer, Irreducible groups of automorphisms of abelian groups . . . . . . . 385

Herbert Stanley Bear, Jr., An abstract potential theory with continuous kernel . . . . 407

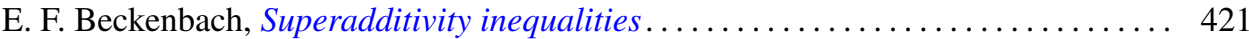

R. H. Bing, The simple connectivity of the sum of two disks . . . . . . . . . . . 439

Herbert Busemann, Length-preserving maps ...................... 457

Heron S. Collins, Characterizations of convolution semigroups of measures . . . . . . 479

Paul F. Conrad, The relationship between the radical of a lattice-ordered group and complete distributivity............................ 493

P. H. Doyle, III, A sufficient condition that an arc in $S^{n}$ be cellular . . . . . . . . . 501

Carl Clifton Faith and Yuzo Utumi, Intrinsic extensions of rings . . . . . . . . . . 505

Watson Bryan Fulks, An approximate Gauss mean value theorem . . . . . . . . . . 513

Arshag Berge Hajian, Strongly recurrent transformations . . . . . . . . . . . . . 517

Morisuke Hasumi and T. P. Srinivasan, Doubly invariant subspaces. II . . . . . . . 525

Lowell A. Hinrichs, Ivan Niven and Charles L. Vanden Eynden, Fields defined by

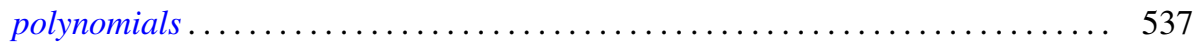

Walter Ball Laffer, I and Henry B. Mann, Decomposition of sets of group

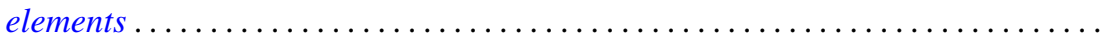

John Albert Lindberg, Jr., Algebraic extensions of commutative Banach

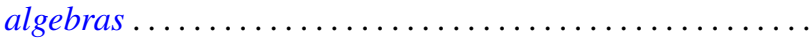

W. Ljunggren, On the Diophantine equation $C x^{2}+D=y^{n} \ldots$

M. Donald MacLaren, Atomic orthocomplemented lattices ....

Moshe Marcus, Transformations of domains in the plane and applications in the

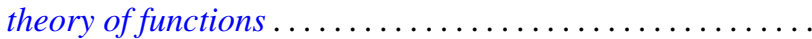

Philip Miles, $B^{*}$ algebra unit ball extremal points . .................. 627

W. F. Newns, On the difference and sum of a basic set of polynomials . . . . . . . 639

Barbara Osofsky, Rings all of whose finitely generated modules are injective ...... 645

Calvin R. Putnam, Toeplitz, matrices and invertibility of Hankel matrices . . . . . . . 651

Shoichiro Sakai, Weakly compact operators on operator algebras . . . . . . . . . 659

James E. Simpson, Nilpotency and spectral operators . . . . . . . . . . . . . 665

Walter Laws Smith, On the elementary renewal theorem for non-identically

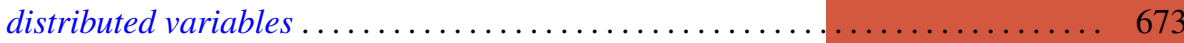

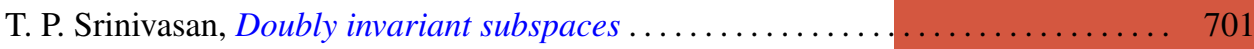

J. Roger Teller, On the extensions of lattice-ordered groups . . . . . . . . . . . . 709

Robert Charles Thompson, Unimodular group matrices with rational integers as

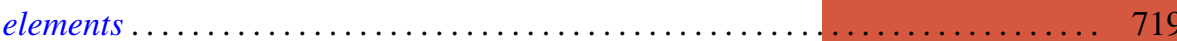

J. L. Walsh and Ambikeshwar Sharma, Least squares and interpolation in roots of unity

Charles Edward Watts, A Jordan-Hölder theorem .................... 731

Kung-Wei Yang, On some finite groups and their cohomology .............. 735

Adil Mohamed Yaqub, On the ring-logic character of certain rings ............ 741

Paul Ruel Young, A note on pseudo-creative sets and cylinders 